\section{Treinamento para disfunção vagal cardíaca com repetições da transição repouso- exercício}

\section{Título em inglês: Training for cardiac vagal dysfunction with repetitions of rest- exercise transition}

\author{
Carlos Vieira Duarte ${ }^{1}$ \\ Claudia Lucia Barros de Castro $^{2}$ \\ Claudio Gil Soares de Araújo ${ }^{1,2}$
}

\begin{abstract}
Resumo
Disfunção vagal cardíaca está associada com um pior prognóstico clínico e um dos potenciais benefícios do exercício físico regular é aumentar o tônus vagal cardíaco (TVC). Como nas transições repouso-exercício-repouso o TVC é rapidamente inibido e reativado, hipotetizou-se que um treinamento repetido desta transição - denominado treinamento vagal (TV) -, em indivíduos com TVC reduzido, poderia reverter essa disfunção. Participantes que ingressaram em um programa de exercício supervisionado (PES) com índice vagal cardíaco (IVC) baixo $(\leq 1,30$ no teste de exercício de quatro segundos) foram randomizados para um estudo com delineamento cruzado (duas etapas de oito semanas), com ou sem três sessões semanais de TV. O TV consistiu de cinco repetições em $5 \mathrm{~s}$ de pedalada rápida sem carga com $55 \mathrm{~s}$ de intervalo em repouso. Após rígidos critérios de inclusão e exclusão (controle de uso de medicação, condição clinica e aderência ao PES) 44 pacientes ( $64 \%$ homens; 65,5 $\pm 11,4$ anos) finalizaram o estudo. Houve discreta melhora no IVC ao final de 16 semanas (1,19 vs 1,22; p=0,02), mas não se pôde afirmar que a diferença no IVC se deveu ao período em que foi realizado o TV ( $p=0,36$ ). Portanto, 16 semanas de PES incluindo oito semanas de TV aumentou a resposta vagal à transição repouso -exercício, embora não tenha sido possível atribuir os resultados exclusivamente ao TV. Futuros estudos são necessários para esclarecer se o TV, com maior número de repetições e/ou período de duração mais longo, poderá promover uma melhora mais acentuada do TVC em indivíduos com níveis iniciais baixos.
\end{abstract}

\section{Palavras-chave}

Sistema nervoso parassimpático; Reabilitação cardíaca; Teste de 4 segundos; Testes autonômicos.

\begin{abstract}
Vagal dysfunction is related to a worse clinical prognosis and one of the potential benefits of regular exercise is to increase cardiac vagal tone (CVT). Since CVT is quickly inhibited and reactivated in the fast rest-exercise-rest transition, it was hypothesized that a training of this transition - called vagal training $(V T)$ - in individuals with reduced CVT could reverse this condition. Patients with low cardiac vagal index (CVI) ( $\leq 1.30$ on the 4-second exercise test) starting a supervised exercise program (SEP) were randomized in a crossover design (two phases, eight weeks each), with or without three weekly VT sessions. VT consisted of five repetitions of sudden, fast unloaded pedaling for five seconds in a cyclergometer, and resting seated for 55 seconds. After applying strict inclusion and exclusion criteria (medication use, clinical condition and adherence to SEP), 44 patients (64\% men and $65.5 \pm 11.4$ years) completed the study. There was a slight improvement in the CVI at the end of 16 weeks (1.19 vs $1.22, p=0.02)$, but it could not be stated this difference was due to the VT ( $p=0.36)$. Therefore, 16 weeks of SEP, including eight weeks of VT training, increased the CVI of those with lower CVI, but this improvement could not be attributed exclusively to the additional VT. Further studies are needed to clarify if more repetitions or a longer period of VT would promote even greater CVT recovery.
\end{abstract}

\section{Keywords}

Parasympathetic nervous system; Cardiac rehabilitation; 4-second exercise test; Autonomic testing.
Rev Bras Ativ Fis Saúde p. 688-697 DOl:

http://dx.doi.org/10.12820/rbafs.v.18n6p688

1 Programa de Pós-graduação em Ciências do Exercício e do Esporte -Universidade Gama Filho - Rio de Janeiro, RJ.

2 Clínica de Medicina do Exercício (CLINIMEX) - Rio de Janeiro, RJ, Brasil. 


\section{INTRODUÇÃO}

Há uma relação inversa e significativa entre o risco de mortalidade e o tônus vagal cardíaco (TVC) ${ }^{1}$, o qual diminui com a idade ${ }^{2}$ e tende a estar reduzido em determinadas condições clínicas ${ }^{3}$, como na doença arterial coronariana ${ }^{4}$, após infarto do miocárdio ${ }^{5}$ e na presença de diabetes melito ${ }^{6}$ de insuficiência cardíaca ${ }^{7}$. Do ponto de vista da saúde pública, faz assim sentido elaborar estratégias para combater essa disfunção, o que talvez seja possível através de intervenções específicas de exercícios físicos, haja visto que sua prática regular tem se mostrado geralmente capaz de provocar uma melhora da modulação autonômica cardíaca, tanto em indivíduos saudáveis do ponto de vista cardiovascular ${ }^{8}$ como em cardiopatas ${ }^{4}$. Por este motivo a influência do treinamento físico na modulação vagal vem sendo objeto de vários outros estudos ${ }^{4-6,9-13}$. Entre as técnicas de avaliação autonômica parassimpática está o teste de exercício de quatro segundos (T4s), que avalia o TVC através da magnitude da variação da frequência cardíaca $(\mathrm{FC})$ na transição rápida repouso -exercício ${ }^{14}$ e que tem se mostrado simples, válido e fidedigno ${ }^{15}$.

Uma possibilidade teórica interessante, e não devidamente explorada na literatura e com potenciais implicações em termos de saúde, seria verificar se um período de treinamento de múltiplas repetições do arco reflexo de inibição e reativação vagal induzidos pelo exercício poderia resultar em benefício específico em indivíduos com disfunção vagal cardíaca. Dados preliminares do nosso grupo de pesquisa, obtidos em participantes de um programa de exercício supervisionado (PES), sugerem que, ao menos em alguns participantes, esse treinamento específico - emulando o protocolo adotado pelo T4s - teve impacto favorável quando feito de forma adicional aos exercícios habitualmente incluídos em um PES. Desta forma, o presente ensaio clínico randomizado objetivou avaliar a possível influência concomitante de oito semanas de treinamento específico da transição rápida repouso-exercício, doravante denominado de treinamento vagal (TV), sobre o TVC em pacientes que iniciaram PES partindo de níveis iniciais baixos de TVC.

\section{MÉTOdOS}

\section{Amostra}

Partindo de um total de 1320 indivíduos que foram submetidos a uma avaliação em Medicina do Exercício em uma clínica privada, entre maio de 2011 e fevereiro de 2013, foram identificados 254 indivíduos com intenção de iniciar um PES. Considerando apenas aqueles com um resultado no T4s igual ou menor que 1,30, foram identificados 177 indivíduos inicialmente aptos a participar no estudo. Os critérios inicialmente aplicados para exclusão dos indivíduos foram: a) possuir um resultado no T4s maior do que 1,30 (caracterizado como um valor limite inferior da normalidade de TVC de acordo com a base de dados do laboratório) na avaliação pré-participação; b) ter marcapasso cardíaco implantado e c) ter limitações osteomioarticulares, cognitivas e/ou neurológicas que interferissem na execução do T4s. Todos os participantes do estudo assinaram um termo de consentimento livre e esclarecido específico e o protocolo do estudo foi aprovado pelo Comitê de Ética em Pesquisa institucional sob o número 161.0.312.000-11, consoante com os termos da Declaração de Helsinque, e com a resolução 196/96 do Conselho Nacional de Saúde. O ensaio clínico está registrado na plataforma virtual Ensaios 
Clínicos (UTN: U1111-1149-5285) e também na base de dados do Clinical Trials sob o número NCT01977742.

A avaliação pré-participação ao PES consistiu em uma anamnese, exame físico, eletrocardiograma e espirometria de repouso, ampla avaliação cineantropométrica, T4s e teste cardiopulmonar de exercício (TCPE) máximo.

\section{Procedimentos}

O TCPE foi realizado em cicloergômetro de membros inferiores (CG-04; Inbrasport; Brasil) seguindo protocolo individualizado de rampa, conforme descrito anteriormente ${ }^{14}$. Para a avaliação do TVC foi utilizado o T4s que, sucintamente, consiste em: 1) sentado no cicloergômetro, realizar uma inspiração rápida e máxima e manter a apneia por 12 segundos; 2) entre o quinto e o nono segundo dessa apneia, pedalar, sem qualquer resistência adicionada, o mais rápido possível; 3) quatro segundos após, retomar ao padrão respiratório normal. A manobra foi sempre realizada pelo menos duas vezes com um intervalo suficiente para que a $\mathrm{FC}$ retornasse a um valor igual ou próximo aquele observado antes da primeira manobra, que por sua vez era próxima da FC de repouso observada no eletrocardiograma de repouso ${ }^{2,14-16}$.

A estimativa do TVC é feita através do resultado do índice vagal cardíaco (IVC) no T4s. O IVC é uma variável adimensional obtida pela razão entre as durações de dois intervalos $\mathrm{RR}$ - o primeiro imediatamente antes do exercício, chamado RRB, e o segundo, o mais curto durante o exercício, chamado $\mathrm{RRC}^{14}$-. O registro eletrocardiográfico foi obtido de forma contínua em uma única derivação, geralmente CM5 ou CC5 (Elite ErgoPC 3.3.6.2, Micromed, Brasil), sendo iniciados quatro segundos antes do primeiro comando verbal e mantidos por 35 segundos. Estas medidas foram realizadas de acordo com o protocolo do T4s, analisadas e reportadas por um avaliador experiente no procedimento. Visando obter a maior resposta vagal do indivíduo, somente a melhor medida obtida foi utilizada para análises posteriores.

Os indivíduos foram alocados de forma randômica em dois grupos, utilizando a função random do software Quattro Pro versão X4: o grupo denominado de ITV iniciava o TV após três sessão de PES, enquanto o outro grupo INTV, somente iniciava o TV após ter completado oito semanas das sessões convencionais do PES. Após oito semanas de PES, as condições eram invertidas: o grupo ITV passava a fazer apenas PES e o grupo INTV iniciava o TV concomitantemente ao PES (figura 1). Inicialmente foram randomicamente alocados 106 indivíduos no grupo ITV e 71 no INTV. Posteriormente, para efeito da análise final dos dados foram eliminados todos os indivíduos que frequentaram menos de 13 sessões em cada uma das duas etapas de oito semanas de realização do estudo, bem como aqueles que alteraram o uso de medicação que pudesse afetar a modulação vagal, que tiveram alteração relevante na condição clinica.

O protocolo de TV era realizado após a breve avaliação médica pré-sessão e antes de iniciar qualquer outro exercício do PES. O TV consistiu em realizar cinco transições rápidas repouso-exercício, da seguinte forma: o indivíduo permaneceu sentado e parado por 55 segundos e em seguida começou a pedalar, o mais rápido possível, por 5 segundos no cicloergômetro de membros inferiores (EC-1600, Cateye, Japão) desligado, de forma a não haver qualquer resistência ao pedalar. Todos os indivíduos foram submetidos a reavaliações com o T4s ao final de cada fase do estudo, isso é, após oito e 16 semanas de intervenção. 


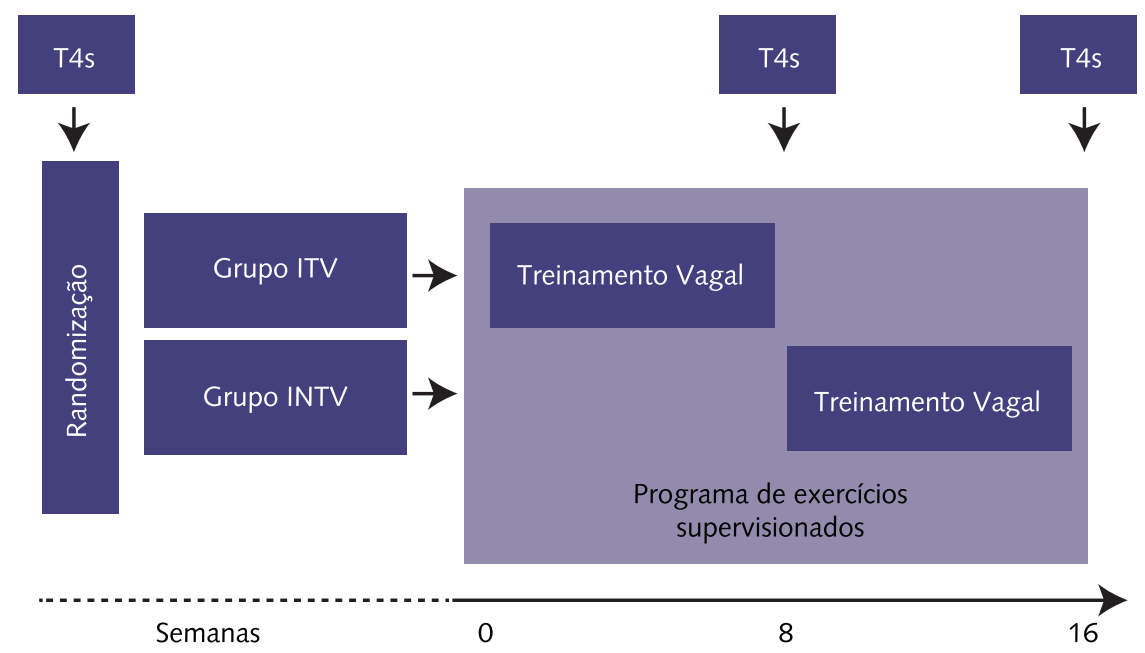

Figura 1 - Desenho do estudo em delineamento cruzado (crossover design): Grupo ITV inicia o Treinamento Vagal na terceira sessão do programa de exercícios supervisionado; Grupo INTV inicia Treinamento Vagal após oito semanas

As sessões de exercício do PES foram compostas por exercícios aeróbios, de força e potência muscular, propriocepção/coordenação motora e flexibilidade, e foram realizadas em ambiente climaticamente controlado sob a presença de médico especializado em Medicina do Exercício e do Esporte, o qual liderava uma equipe multiprofissional, integrada por educadores físicos, fisioterapeutas e profissionais de enfermagem. Ao início da sessão, o peso corporal foi medido e o frequencímetro (Polar, Finlândia) colocado; em seguida, o médico registrava a FC e a pressão arterial de repouso, (tensiômetro digital Omron XEL-907, Omron, Estados Unidos), e prescreveu o componente aeróbio da sessão, baseado nas recomendações existentes no laudo da última avaliação, nas condições do paciente no dia e nos dados colhidos na sessão anterior. A parte aeróbia da sessão de exercício durou entre 30 e 40 minutos, objetivando-se alcançar a zona-alvo de FC caracterizada a partir do TCPE, mesclando atividades em modo constante ou intervalado, em esteiras rolantes, cicloergômetros de membros superiores e inferiores e remoergômetros. Em seguida ocorreu a parte não aeróbia, prescrita e supervisionada pelos educadores físicos e fisioterapeutas, primariamente voltados para o aprimoramento da força/potência muscular, da flexibilidade e do equilíbrio. As prescrições dos exercícios foram frequentemente revisadas.

\section{Análise estatística}

O cálculo para o tamanho da amostra com $\alpha=0,05$ e $\beta=0,80$ para detectar uma diferença de 0,05 no IVC, considerando um desvio padrão de 0,10 , resultou na necessidade de uma amostra de pelo menos 18 indivíduos (Primer of Biostatistics, versão 4, Reino Unido), portanto bastante abaixo do número final de casos do presente estudo. Para analisar o efeito no IVC de 16 semanas de PES no qual se fez apenas concomitantemente oito semanas de TV - nas primeiras oito ou nas últimas oito semanas -, utilizou-se o teste-t emparelhado. A normalidade da distribuição dos resultados do IVC inicial, para os dois grupos, foi confirmada pelo teste de D'Agostino Pearson, havendo também homocedasticidade quando as variâncias foram comparadas pelo teste-F. Para tentar evidenciar o efeito isolado do TV, utilizou-se análise estatística específica ${ }^{17}$, a qual avalia a diferença entre os períodos (apenas PES ou PES + TV) através de teste-t simples para amostras 
independentes, usando as diferenças intraindividuais entre os desfechos em ambos os períodos como dado bruto.

Como em um estudo com delineamento cruzado (crossover trial) o indivíduo é o controle de si próprio, não há necessidade formal de um grupo controle adicional para se observar a resposta do TVC especificamente em relação ao TV. Entretanto, como é possível que o PES por si só já aumente o TVC mesmo se realizado sem o TV e como dispomos de dados relativos ao IVC de clientes que frequentaram o PES em época anterior à implementação do TV, optamos por analisar também a evolução do IVC de 15 indivíduos que fizeram somente o PES (reavaliados entre quatro e 12 meses) - doravante denominado como grupo controle. Sendo assim, foi possível analisar o comportamento do IVC em um grupo distinto e que não realizou o protocolo do TV e comparar com a amostra final do presente estudo.

Em síntese, foram feitas as seguintes análises: a) teste-t entre as médias do IVC pré e pós PES do grupo controle de 15 indivíduos que realizaram o PES, mas não o TV, para se observar o efeito isolado do PES no TVC; b) teste-t emparelhado da amostra inteira do presente estudo $(n=44)$ para avaliar o efeito de 16 semanas de PES, incluindo oito semanas de TV, comparando as médias antes e após as 16 semanas (independentemente de ter sido feito TV nas oito semanas iniciais ou finais do estudo); e c) para avaliar o efeito específico e adicional do TV no IVC, utilizou-se teste-t simples usando a diferença intra-indivíduo após cada um dos dois períodos (oito semanas fazendo TV ou oito semanas sem fazer o TV) como dado bruto. Para esta análise faz-se um pré-teste que avalia se, entre uma fase e outra, há um possível efeito residual (influência da passagem do tempo entre as fases e independente do tratamento); também através de teste-t independente, mas utilizando a soma e não a diferença dos valores medidos em ambos os períodos para cada indivíduo e comparado sobre os dois grupos ${ }^{17}$.

\section{RESULTADOS}

Dos 177 indivíduos que se propuseram a iniciar o PES, apenas 164 efetivamente ingressaram. Após eliminar os indivíduos de acordo com a frequência mínima (13 sessões em cada uma das etapas de oito semanas) e os demais critérios de exclusão, obtivemos uma amostra final de 44 indivíduos, sendo 31 no grupo ITV e 13 no grupo INTV. Os principais dados antropométricos e demográficos desses 44 indivíduos que concluíram o estudo são apresentados na tabela 1. Estes dados são bastante similares aos do grupo controle de 15 indivíduos que participaram exclusivamente de PES, mas sem TV, a saber: $60 \%$ de homens com (medias \pm desvio padrão) $57 \pm 10,2$ anos de idade, $79 \pm 12,4 \mathrm{~kg}$ de peso corporal, $169 \pm 7,6 \mathrm{~cm}$ de altura, IVC de 1,31 $\pm 0,17$ e FC de repouso de $70 \pm 11$ batimentos por minuto.

Os resultados indicam que: a) o IVC inicial no grupo controle não diferiu do IVC após o período participando do PES ( $\mathrm{n}=15$; média \pm erro padrão da média; $1,31 \pm 0,05$ vs $1,25 \pm 0,03, \mathrm{p}=0,06$ ); b) 44 pacientes submetidos ao presente estudo - 16 semanas de PES e 8 semanas de TV - tiveram um discreto mas significativo aumento do IVC ( $1,19 \pm 0,01$ vs $1,22 \pm 0,02, \mathrm{p}=0,02)$ (figura 2$)$; e c) embora não tenha havido no pré-teste efeito residual entre as fases $(\mathrm{p}=0,41)$, o que possibilita a comparação entre os períodos com e sem TV, não se pôde atribuir exclusivamente ao TV a melhora discreta no IVC após 16 semanas de PES e oito semanas de TV ( $\mathrm{p}=0,36)$. 
Tabela 1 - Principais dados antropométricos e demográficos da amostra.

\section{Variáveis}

Idade (anos)

Altura (cm)

Peso corporal $(\mathrm{kg})$

Índice de massa corporal $\left(\mathrm{kg} / \mathrm{m}^{2}\right)$

Índice vagal cardíaco (ms)

Intervalo RR mais longo - RRB (ms)

Intervalo RR mais curto - RRC (ms)

Frequência cardíaca de repouso (bpm)
Resultados

$65,5 \pm 11,4(39-83)$

$168,5 \pm 9,9(149-192)$

$79,4 \pm 16,3(47-117)$

$27,8 \pm 4,4(19-38)$

$1,19 \pm 0,07(1,04-1,30)$

$885 \pm 140(650-1190)$

$744 \pm 119(500-950)$

$64 \pm 9,4(45-88)$

${ }^{\text {* }}$ resultados expressos como média \pm desvio padrão (mínimo e máximo)

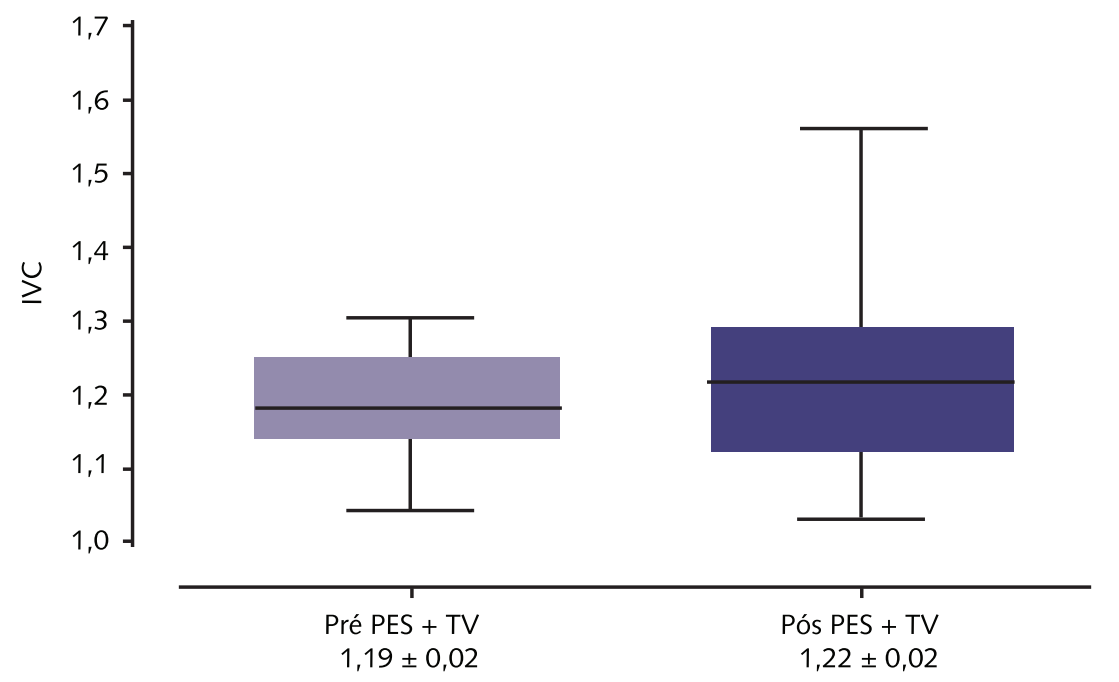

Figura 2 - Efeito do programa de exercícios supervisonado (PES) juntamente com o treinamento vagal (TV) no índice vagal cardíaco (IVC) após 16 semanas (8 semanas de TV nas primeiras ou últimas oito semanas); $p=0,02$.

\section{DISCUSSÃO}

A principal informação deste estudo é que um aumento no TVC (representado pelo IVC) pode ocorrer após 16 semanas de participação em um PES, quando for realizado concomitantemente um TV por, pelo menos, oito dessas semanas. Isso contrasta com a observação de um patamar inalterado a minimamente reduzido de TVC em uma análise retrospectiva de pacientes que haviam participado do PES sem a inclusão do TV. Observou-se adicionalmente que, embora o TV pareça influenciar positivamente a adaptação no IVC, o delineamento cruzado não permitiu afirmar que o TV foi o responsável pela discreta melhora no IVC.

A melhora na modulação autonômica em decorrência do treinamento físico tem sido objeto de diversos estudos, com diferentes intervenções, durações, amostras e, até certo ponto, resultados variados. Entende-se de maneira geral que o trei- 
namento físico altera o balanço autonômico a favor de uma predominância vagal ${ }^{18}$, o que tem implicações importantes para indivíduos com delicada condição clínica, como aqueles com insuficiência cardíaca ${ }^{7}$ ou pós-infarto do miocárdio 5 . Por outro lado, analisando alguns destes estudos e utilizando como base a elegante revisão sobre os efeitos do treinamento redigida por Routledge $e t$ a ${ }^{11}$, notam-se algumas evidências com resultados distintos.

Assim como é possível notar a melhora em índices da variabilidade da FC relacionados ao TVC através do treinamento físico realizado por oito semanas após um infarto agudo do miocárdio ${ }^{12}$ ou por três meses em indivíduos com insuficiência cardíaca ${ }^{19}$, da mesma forma destacam-se evidências com resultados discordantes, tais como: ausência de diferença entre grupos controle e exercício no componente de alta frequência da variabilidade da FC (no período entre o segundo e o oitavo mês) após infarto do miocárdio ${ }^{20}$; ausência de diferença na modulação autonômica em repouso (supino, livre respiração) entre grupo controle e exercício após três meses de reabilitação cardíaca de baixa intensidade, em homens e mulheres com insuficiência cardíaca (aumento apenas na reatividade vagal) ${ }^{21}$, ausência de melhora na variabilidade da FC de homens diabéticos após um ano de treinamento, com duas sessões semanais de treinamento aeróbio e duas sessões semanais de treinamento de força ${ }^{6}$ e ausência de melhora nos índices de variabilidade da FC. Isto reforça a ideia de que o entendimento dos principais mecanismos e adaptações autonômicas possíveis de serem obtidos pelo treinamento físico ainda não estão completamente esclarecidos e reforça a necessidade de serem desenvolvidos e testados protocolos alternativos, tal como o TV utilizado no presente estudo.

Além dos benefícios obtidos com o treinamento tradicional, caracterizado por um período maior de intervenção e sessões mais longas, é possível que intervenções mais curtas possam também influir positivamente no TVC. La Rovere et a $\ell^{2}$. demonstraram que, mesmo com um programa de treinamento mais curto (quatro semanas), já é possível obter algum grau de alterações favoráveis no TVC, ainda que somente tivesse sido demonstrado como resposta à um único tipo de estresse fisiológico - tilt test -, mas não em situação de repouso. É possível que o benefício do treinamento aeróbio sobre a modulação autonômica seja menor do que influência observada na potência aeróbia máxima, conforme é exemplificado pelo aumento de 6,4\% no consumo máximo de oxigênio com treinamento moderado (duas a três vezes por semana) em homens sedentários entre 55 e 75 anos de idade, que não tiveram sua modulação autonômica cardíaca alterada ${ }^{13}$.

Independentemente da importante influência da genética na variabilidade da FC, estimada entre 39 e $65 \%^{23}$, a adaptação vagal ao treinamento tem sido estudada por diferentes ângulos, entre eles a duração da sessão de exercício. Estudos experimentais apontam uma adaptação vagal com sessões diárias de 30 minutos, sem benefícios adicionais ao se realizar sessões com o dobro de duração ${ }^{10}$. Neste aspecto, o presente estudo testou a hipótese de um treinamento de curta duração (5 minutos/3x semana/8 semanas), realizado juntamente ao treinamento físico aeróbio e de fortalecimento muscular convencional, ter um impacto positivo no TVC, o que se confirmou, ainda que de forma modesta.

Diversos estudos do nosso grupo, iniciados nos anos $80^{24} \mathrm{e}$, logo a seguir, com o T4s (método utilizado neste estudo) investigaram a modulação vagal e as respostas da FC nas transições repouso-exercício-repouso. O T4s foi validado farmacologicamente ${ }^{14} \mathrm{e}$ vem sendo utilizado por outros grupos de pesquisa, nacionais $\mathrm{s}^{25} \mathrm{e}$ internacionais ${ }^{26}$. O protocolo do TV se baseia na inibição vagal induzida pelo exercício e esta não parece ser influenciada pela manobra respiratória que é realizada no 
$\mathrm{T} 4 \mathrm{~s}^{16}$, mas não no TV. Isto reforça a ideia de que houve, de fato, a retirada do vago em cada uma das transições repouso-exercício-repouso realizadas no TV.

Em relação à segurança do TV, durante o período do estudo - compreendendo a pouco mais de 900 sessões de TV - houve apenas um único episódio no qual o pedal do ciclo de membros inferiores se soltou, apesar do uso de pedaleiras, durante o pedalar rápido, ferindo superficialmente a perna de um dos indivíduos do estudo. Excluindo este acidente, todos os indivíduos realizaram o protocolo do TV sem complicações, mesmo aqueles em idade avançada ( $25 \%$ da amostra com mais de 75 anos de idade). Em adendo, o TV mostrou-se, em geral, simples, bem aceito pelos indivíduos, bastante seguro e fácil de fazer, uma vez que até indivíduos de mais de 80 anos conseguiram cumprir ambas as etapas (treinando um total de pelo menos 26 sessões) de forma simples e sem intercorrências relevantes.

Ao melhor do nosso conhecimento, não há na literatura um protocolo específico de treinamento físico para aumentar o TVC. A tentativa de estabelecer isto, especialmente em um ambiente com alta validade externa (um PES) e utilizando um protocolo curto (cinco minutos), simples (não demanda equipamentos sofisticados), de baixa demanda energética (pedalar rapidamente, em um ciclo sem carga), bastante seguro (um único evento desfavorável de baixa complexidade em 908 sessões), é provavelmente um dos aspectos positivos do estudo.

Dentre as possíveis limitações do estudo estão o fato de que sete indivíduos fizeram por períodos variados de tempo durante as 16 semanas de intervenção, de modo concomitante, um protocolo de treinamento de preensão manual ${ }^{27}$, algo que pode afetar a modulação autonômica ${ }^{28}$. Contudo, em análise separada, não se evidenciou diferença na média da magnitude do ganho no IVC entre os que participaram e aqueles que não participaram do protocolo de preensão manual (delta IVC $=0,05$ vs 0,$03 ; p=0,49)$. Também é possível que um período maior que oito semanas para o TV fosse interessante, mas isto provavelmente afetaria de maneira relevante a aderência, o que poderia ter comprometido de forma importante o estudo. Finalmente, se fosse possível eliminar a influência dos exercícios aeróbios e de fortalecimento, alocando os indivíduos para fazer exclusivamente o TV, é provável que os resultados fossem mais específicos, mas pelo fato do estudo ter se desenvolvido dentro do contexto de um PES realizado em uma clínica privada (contribuindo para uma amostra de 44 indivíduos após quase dois anos de coleta de dados), isto não seria provavelmente viável nem ético.

Outra potencial limitação é o fato do IVC dos 15 indivíduos do grupo controle ser mais alto que o dos 44 indivíduos do grupo PES + TV. Uma vez que este grupo serviu apenas para ilustrar que, em indivíduos frequentando um PES e que não fazia o TV, o IVC não aumenta, pode-se supor que o TV contribuiu na melhora do TVC no período de 16 semanas. Um ponto forte do estudo é o grande número de indivíduos incluídos no ensaio clínico randomizado, que, apesar da grande taxa de abandono (algo esperado para um estudo longitudinal, utilizando treinamento físico, que não permitiu ausência prolongada e foi desenvolvido em um serviço particular), ainda assim obteve um número representativo de indivíduos, na realidade, maior do que a maioria dos estudos realizados em condições similares, e de acordo com o cálculo amostral.

Futuros estudos se fazem necessários para entender possíveis implicações de um treinamento com repetições da transição rápida repouso-exercício, variando número de séries, tempo de intervenção e o uso de diferentes ergômetros. No mesmo contexto é possível que haja uma considerável variabilidade na adaptação intra-individual do TVC ao TV, talvez influenciada por aspectos clínicos, por 
exemplo, região de comprometimento fibrótico miocárdico ${ }^{29}$, como observado em estudos clínicos, ou presença de disfunção ventricular esquerda, observado em estudos experimentais ${ }^{30}$.

Conclui-se então que o PES, juntamente com o TV, parece ser uma opção eficaz para aumentar o TVC, ainda que os efeitos sejam discretos e não se possa afirmar que o TV seja exclusivamente responsável pelo aumento no IVC. Dessa forma, futuros estudos são necessários para esclarecer mais detalhes sobre este tipo de intervenção, como esclarecer se uma maior duração e/ou maior número de repetições no TV poderia contribuir para a elevação dos níveis de TVC em indivíduos com níveis iniciais baixos.

\section{Agradecimentos}

Os autores agradecem ao CNPq e FAPERJ pelo suporte financeiro concedido.

\section{REFERÊNCIAS}

1. Kleiger RE, Miller JP, Bigger JT,Jr, Moss AJ. Decreased heart rate variability and its association with increased mortality after acute myocardial infarction. Am J Cardiol 1987;59:(4);256-62.

2. Duarte CV, Araujo CG. Cardiac vagal index does not explain age-independent maximal heart rate. Int J Sports Med 2013;34:(6);502-6.

3. Lahiri MK, Kannankeril PJ, Goldberger JJ. Assessment of autonomic function in cardiovascular disease: physiological basis and prognostic implications.J Am Coll Cardiol 2008;51:(18);1725-33.

4. Lucini D, Milani RV, Costantino G, Lavie CJ, Porta A, Pagani M. Effects of cardiac rehabilitation and exercise training on autonomic regulation in patients with coronary artery disease. Am Heart J 2002;143:(6);977-83.

5. Martinez DG, Nicolau JC, Lage RL, Toschi-Dias E, Matos LD, Alves MJ, et al. Effects of longterm exercise training on autonomic control in myocardial infarction patients. Hypertension 2011;58:(6);1049-56.

6. Loimaala A, Huikuri HV, Koobi T, Rinne M, Nenonen A, Vuori I. Exercise training improves baroreflex sensitivity in type 2 diabetes. Diabetes 2003;52:(7);1837-42.

7. Coats AJ, Adamopoulos S, Radaelli A, McCance A, Meyer TE, Bernardi L, et al. Controlled trial of physical training in chronic heart failure. Exercise performance, hemodynamics, ventilation, and autonomic function. Circulation 1992;85:(6);2119-31.

8. Sandercock GR, Bromley PD, Brodie DA. Effects of exercise on heart rate variability: inferences from meta-analysis. Med Sci Sports Exerc 2005;37:(3);433-9.

9. Paschoal MA, Pinheiro TT, Brigliador GM, Caruso TMA, Lima LNG. Efeito do treinamento físico aeróbio sobre a reativação vagal cardíaca em jovens sedentárias. Rev Bras Ativ Fis e Saúde 2012;17:(5);403-13.

10. Sant'Ana JE, Pereira MG, Dias da Silva VJ, Dambros C, Costa-Neto CM, Souza HC. Effect of the duration of daily aerobic physical training on cardiac autonomic adaptations. Auton Neurosci 2011;159:(1-2);32-7.

11. Routledge FS, Campbell TS, McFetridge-Durdle JA, Bacon SL. Improvements in heart rate variability with exercise therapy. Can J Cardiol 2010;26:(6);303-12.

12. Sandercock GR, Grocott-Mason R, Brodie DA. Changes in short-term measures of heart rate variability after eight weeks of cardiac rehabilitation. Clin Auton Res 2007;17:(1);39-45.

13. Verheyden B, Eijnde BO, Beckers F, Vanhees L, Aubert AE. Low-dose exercise training does not influence cardiac autonomic control in healthy sedentary men aged 55-75 years. J Sports Sci 2006;24:(11);1137-47.

14. Araujo CGS, Castro CLB, Nobrega ACL. Heart rate responses to deep breathing and 4-seconds of exercise before and after pharmacological blockade with atropine and propranolol. Clin Auton Res 1992;2:(1);35-40.

15. Araújo CGS, Ricardo DR, Almeida MB. Fidedignidade intra e interdias do teste de exercício de 4 segundos. Rev Bras Med Esporte 2003;9:(5);293-8. 
16. Furtado EC, Ramos PS, Oliveira RB, Araújo CG. Influência da padronização respiratória na resposta da frequência cardíaca à transição repouso-exercício em coronariopatas de um programa de exercício supervisionado. Rev SOCERJ 2009;22:(2);86-92.

17. Wellek S, Blettner M. On the proper use of the crossover design in clinical trials: part 18 of a series on evaluation of scientific publications. Dtsch Arzteb1 Int 2012;109:(15);276-81.

18. Shi X, Stevens GH, Foresman BH, Stern SA, Raven PB. Autonomic nervous system control of the heart: endurance exercise training. Med Sci Sports Exerc 1995;27:(10);1406-13.

19. Kiilavuori K, Toivonen L, Naveri H, Leinonen H. Reversal of autonomic derangements by physical training in chronic heart failure assessed by heart rate variability. Eur Heart J 1995;16:(4);490-5.

20. Mazzuero G, Lanfranchi P, Colombo R, Giannuzzi P, Giordano A. Long-term adaptation of 24-h heart rate variability after myocardial infarction. The EAMI Study Group. Exercise Training in Anterior Myocardial Infarction. Chest 1992;101:(5 Suppl);304S-8S.

21. Malfatto G, Branzi G, Riva B, Sala L, Leonetti G, Facchini M. Recovery of cardiac autonomic responsiveness with low-intensity physical training in patients with chronic heart failure. Eur J Heart Fail 2002;4:(2);159-66.

22. La Rovere MT, Mortara A, Sandrone G, Lombardi F. Autonomic nervous system adaptations to short-term exercise training. Chest 1992;101:(5 Suppl);299S-303S.

23. Busjahn A, Voss A, Knoblauch H, Knoblauch M, Jeschke E, Wessel N, et al. Angiotensinconverting enzyme and angiotensinogen gene polymorphisms and heart rate variability in twins. Am J Cardiol 1998;81:(6);755-60.

24. Araujo CG. Fast “ON" and "OFF" heart rate transients at different bicycle exercise levels. Int J Sports Med 1985;6:(2);68-73.

25. Pimentel AS, Alves ES, Alvim RO, Nunes RT, Costa CMA, Lovisi JCM, et al. Polar S810 como recurso alternativo ao eletrocardiograma no teste de exercício de 4 segundos. Arq Bras Cardiol 2010;94580-4.

26. Knopfli BH, Bar-Or O. Vagal activity and airway response to ipratropium bromide before and after exercise in ambient and cold conditions in healthy cross-country runners. Clin J Sport Med 1999;9:(3);170-6.

27. Araujo CG, Duarte CV, Goncalves FA, Medeiros HB, Lemos FA, Gouvea AL. Hemodynamic responses to an isometric handgrip training protocol. Arq Bras Cardiol 2011;97:(5);413-9.

28. Millar PJ, MacDonald MJ, Bray SR, McCartney N. Isometric handgrip exercise improves acute neurocardiac regulation. Eur J Appl Physiol 2009;107:(5);509-15.

29. Singh N, Mironov D, Armstrong PW, Ross AM, Langer A. Heart rate variability assessment early after acute myocardial infarction. Pathophysiological and prognostic correlates. GUSTO ECG Substudy Investigators. Global Utilization of Streptokinase and TPA for Occluded Arteries. Circulation 1996;93:(7);1388-95.

30. Kinugawa T, Dibner-Dunlap ME. Altered vagal and sympathetic control of heart rate in left ventricular dysfunction and heart failure. Am J Physiol 1995;268:(2 Pt 2);R310-16.

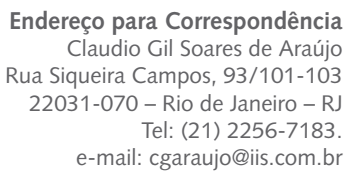

